\title{
Dynamic inter-fibre failure of unidirectional composite laminates with through-thickness reinforcement
}

\author{
Hao Cui a,b,1 , Mehdi Yasaee a, António R. Melro ${ }^{c}$ \\ a School of Aeronautics, Transport and Manufacturing, Cranfield University \\ ${ }^{\mathrm{b}}$ Department of Engineering Science, University of Oxford \\ ${ }^{\mathrm{C}}$ Bristol Research Institute, ACCIS, University of Bristol, Bristol, UK
}

\begin{abstract}
Unidirectional composite laminates reinforced in the thickness direction with Z-pins are tested in tension and compression with different off-axis angles at high loading rate. A split Hopkinson bar setup and a high speed imaging system have been used for these dynamic tests. It is found that the inter-fibre strength was reduced by Z-pinning in tension and shear dominated failure modes, and the reduction from dynamic loading conditions is greater than that in quasi-static. However, the laminate strength was not significantly influenced by Zpinning when loaded predominantly in compression. This difference was caused by the transition of failure mechanisms around Z-pins. The stiffness of the laminate was not affected by Z-pins at elevated strain rates, since the increased epoxy stiffness helped to make up for the stiffness loss due to reduced fibre volume fraction resulting from Z-pins.
\end{abstract}

\section{Key words}

${ }^{1}$ Corresponding author: hao.cui@cranfield.ac.uk 


\section{Introduction}

Longitudinal carbon fibre reinforced composite laminates are widely used in aerospace and many industrial sectors. The inter-fibre failure modes, such as inter-laminar delamination and intra-laminar cracking, are some of the dominating failure modes for these laminated composites [1]. The strength and resistance against these failure modes can be improved by toughening the polymer matrix with nanoparticles [2,3] or nanofibers [4] or introducing through thickness reinforcement (TTR). The latter method has been researched extensively and shown to be quite effective at mitigating the inter-laminar delamination damage [5-7]. Z-pinning has been one of the most popular solutions of TTR; where metallic or carbon fibre rods are inserted through the laminate thickness for resisting the growth of delamination [7].

Z-pinning significantly increases the resistance against the inter-laminar crack propagation[8-11] and has recently been found to be effective in improving the dynamic delamination toughness, although its efficiency decreases with loading rate[12,13]. The outof-plane performance improvement comes at a cost of the in-plane performance, which will see reduction in strength due to the defects caused by the Z-pinning process.

Z-pins are normally inserted into uncured preform made of prepregs, see $[14,15]$ for details. The microstructures of laminates around Z-pins are then changed generating considerable fibre waviness and deflection [16]. There are resin-rich pockets formed around Z-pins, which reduce the average fibre volume fraction [17]. As a result of these microstructural changes, the in-plane stiffness and strength will inevitably be influenced. The longitudinal modulus was reported to be reduced by more than $10 \%$ with Z-pinning [18], the waviness of fibres contributes to premature failure[16], and the longitudinal strength was 
reduced both in tension [15] and compression modes [16,19]. Compared to research on the fibre dominated failure modes, there is limited work studying the matrix dominated interfibre failure response. Systematic study on the mechanical behaviour of Z-pinned laminates in combined compression and shear load has been conducted by the authors recently [17]. It was shown that the Z-pinned laminate stiffness and the strength of shear dominated failure modes is significantly reduced. Interestingly, improvements in the compression dominated failure modes were observed with the addition of Z-pins [17].

Many composite structures may be threatened by impact loading [20], and comprehensive understanding about the dynamic property of Z-pinned laminates is vital for the design and analysis of such structures $[12,13,21]$. To the authors' knowledge, there is no research reported addressing the dynamic in-plane failure process of Z-pinned composites yet. The resin matrix and interface between fibre and matrix has been reported to be very sensitive to strain rate [22]. Considering the presence of resin pockets and complicated interaction between laminates and Z-pins discovered recently [17], the inter-laminar failure process under dynamic loading condition may be different from that in quasi-static case.

The paper aims to investigate the influence of Z-pins and strain rate, on the inter-fibre failure of unidirectional composite laminates, under different stress spectrums including tension, compression and tension/compression coupled with in-plane shear. The dynamic inter-fibre failure properties of Z-pinned laminates is characterized using the split Hopkinson bar system for the first time. The specimen and test setup are introduced in the next section, followed by the experimental results and corresponding discussions. Brief conclusions are outlined in the last section. 


\section{Experiments}

\section{Sample preparation}

Off-axis loading on unidirectional laminates results in combined transverse normal stress and in-plane shear stress, and the ratio between these two stress components can be controlled by tuning the off-axis angle[23,24]. In this work, two unidirectional laminates were prepared with IM7/8552 prepregs with 40 plies and nominal thickness of $5 \mathrm{~mm}$. One of the laminates was reinforced with T300/BMI Z-pins with a pin interval of $1.75 \mathrm{~mm}$. For Z-pins of $0.28 \mathrm{~mm}$ in diameter this results in a $2 \%$ volume fraction in the laminates.

Off-axis compression and tension samples were then cut from unidirectional laminates as illustrated in Fig.1. The edge of samples was grinded to make the dog bone shape of tension samples and to ensure the alignment of top and bottom surface of compression samples. Tension samples were inserted into screwed end caps, for connection with testing machine. Four different angles were selected for compression samples between fibre orientation and loading axis: $30^{\circ}, 45^{\circ}, 60^{\circ}$, and $90^{\circ}$; two different angles were selected for tension samples: $45^{\circ}$ and $90^{\circ}$. These samples cover a relatively broad spectrum of transverse tension/compression stress and in-plane shear stress. Unpinned samples were prepared as well, to be used as reference.

Surface of samples was painted with white paint using air brush, and then black spackles were painted on top to facilitate the digital image correlation (DIC) process.

\section{Test and data process}

All experiments have been conducted in both quasi-static and dynamic loading rate. The standard Zwick Roell 250 test machine has been used for the quasi-static tests. Detailed 
test setup may be referred to [17]. The split Hopkinson tension/compression bars were employed for dynamic experiments, both tension and compression bars are shown in Fig.2a\&b. The striker is accelerated with compressed gas to a certain velocity, and then hit the end of loading bar, resulting in an impact pulse. This impact pulse travels along the loading bar and the input bar, and then applies tension/compression load on the sample during its transmission to the output bar. The impact pulse can be measured with strain gauges mounted on both the input bar and the output bar, and recorded with oscilloscope at sampling rate higher than $10 \mathrm{MHz}$. The velocity of striker ranged from 4 to $10 \mathrm{~m} / \mathrm{s}$, resulting in nominal strain rate of around $300 \mathrm{~s}^{-1}$.

A complete test system is shown in Fig.2c.The ultra-high speed Kirana camera was used for recording the deformation and failure process during experiments. Typical frame rate used in these experiments was around 200,000 frames per second. All images from Kirana camera were analysed with DIC provided by GOM Aramis software. The true axial strain $\varepsilon_{x x}$ is determined from the measured displacement within the gauge section of the sample.

In quasi-static compression experiments, the normal strain is distributed uniformly across the whole sample. The strain can then be analysed by the relative displacement on both ends of the sample, which can be monitored using a laser extensometer. For dynamic tests, the deformation $\delta$ can be calculated using the measured displacement on the end of both input and output bars. The bar displacement is calculated from the strain gauge signal using 1D wave theory for split Hopkinson bar [25]. The dog bone shape was employed in the tension samples, and only the rectangular section in the centre (see Fig.1c) can provide uniform distribution of the stress and strain. The deformation of this gauge section in tension 
tests was analysed from camera images using DIC method. For the prismatic shape used in compression tests, the displacement from SHPB theory can be used for the calculation of strain.

The force, $F$, was obtained with strain gauge signal [25] in all compression dynamic experiments, which is used to calculate the axial stress data knowing the sample cross-section area.

\section{Results and discussions}

\section{Failure modes}

There are three different inter-fibre failure modes as defined by Puck [1], each of them corresponding to a certain range of combination between the transverse compression and in plane shear, as sketched in Fig.3. Previous work at quasi-static loading rate has demonstrated the different influence of Z-pins on inter-fibre failure process, depending on the ratio between compressive transverse stress $\left(\sigma_{22}\right)$ and in-plane shear stress $\left(\tau_{12}\right)$ [17]. This section aims to show the representative fracture of Z-pinned laminates in all these three inter fibre failure modes and highlight the influence of Z-pinning by comparison with unpinned samples.

Mode " $A$ " failure is caused by a combined stress of transverse tension and in-plane shear, which is represented here by the dynamic tension test with off-axis angle of $45^{\circ}$, as shown in Fig.4a\&b. Since all cracks were vertical in the sample thickness direction, only the in-plane view of the fracture process was monitored. Both unpinned and Z-pinned samples had very similar fracture behaviour, where limited number of cracks parallel to fibre direction resulted in the complete failure of samples. Dynamic compression tests with off-axis angle of $30^{\circ}$ is shown in Fig.4c\&d, for representing the Mode " $\mathrm{B}$ " failure that is shear stress dominated 
with moderate transverse compression stress. In both Mode " $A$ " and " $B$ ", the influence of Zpinning on the fracture is insignificant from these high-speed camera images.

A mode " $C$ " failure is represented by the dynamic transverse compression failure. Since inclined cracks with respect to the sample thickness direction were expected in such failure mode, side view images from Kirana camera are shown in Fig.5 for both unpinned and Z-pinned samples. Cracks initiated near the edges in both cases, which then propagated at an angle that is in agreement with the Puck's inter-fibre failure theory $[1,17,26]$. The dominating crack in unpinned sample propagated along a relatively constant direction until complete failure. In the Z-pinned samples, the dominating crack was retarded and branched in different directions. Complex crack paths were formed in Z-pinned samples, compared to the relatively simple crack plane of unpinned ones.

\section{Stress-strain response}

The displacement field of the samples was obtained using two different methods as introduced previously: the displacement calculated from strain signal on the bars and the displacement analysed from camera images using DIC. A comparison of these two methods is conducted for one $45^{\circ}$ off-axial dynamic compression sample, as shown in Fig.6. The stressstrain curves from both methods are consistent with each other. The last part of the section was not successfully captured with DIC due to the fact that the system is no longer valid in areas with large deformation and cracking. In this work, all stress-strain data for compression tests was obtained from the strain signal using split Hopkinson bar theory [27], while for the tension experiments, the displacement field was processed with DIC.

Typical stress-strain curves from all different test conditions are summarized in Fig.7. Stress initially increased in a linear manner with strain in all cases, which is followed by some 
nonlinearity on the curves largely due to the plasticity of epoxy matrix. The high strain rate (HR) in dynamic experiments have delayed the occurring of this nonlinearity. It is very significant when loaded in compression, especially for the shear dominated cases with offaxis angle of $30^{\circ}$ and $45^{\circ}$. Such behaviour is largely caused by the enhanced yielding stress of the investigated materials at high loading rate [22].

The tension test data in Fig.7e) and f) showed that it can be very challenging to measure the transverse tension properties of unidirectional material. Although a decent linear elastic behaviour is reproduced in all quasi-static cases, the dynamic tension data has been predominantly scattered. The displacement here was very small before failure; less than $100 \mu \mathrm{m}$ over the gauge section. A tiny degree of misalignment (less than $0.05^{\circ}$ ) of the tension sample with respect to loading axis can result in artificial decrease/increase of the apparent displacement that is comparable with the true amount of deformation. The input bar and output bar may not be strictly co-axial in practice, therefore misalignment of samples can be inevitable. The dynamic tension tests should be improved in future for better testing quality.

The stiffness of the samples was extracted from the linear elastic stage of the stressstrain curves, and is shown in Fig.8. Due to high scatter in dynamic tension tests, only compression test data is shown. In contrast to the quasi-static experiment where Z-pinning has reduced the stiffness for all off-axis angles [17], the dynamic test didn't show any clear influence for Z-pinned laminates. The stiffness of unpinned samples also seemed to be consistent regardless of loading rate for each off-axis angle, despite the scattered data in $30^{\circ}$ off-axis experiments. The distribution of normal strain along loading direction obtained with DIC is shown in Fig.9, for Z-pinned transverse compression tests at both quasi-static and high loading rate. The strain difference around Z-pins is clearly noticeable from the quasi-static 
test (Fig.9a) due to the epoxy pockets around pins having lower modulus and yielding stress than the laminates. However, in dynamic tests, the strain distribution across the whole sample didn't suggest any correlation with the Z-pins. Previous studies have reported the increase in the stiffness of epoxies with strain rate [22], meaning the epoxy rich resin pockets with increased stiffness may be responsible for the increased stiffness of full Z-pinned samples.

The strain corresponding to critical failure stress is presented in Fig.10a. For the shear dominated compression tests with off-axis angle of $30^{\circ}$ and $45^{\circ}$, the failure strain has been considerably reduced by the Z-pins at high loading rate. Although scatters have been noticed in the strain value measured in dynamic tension tests, the quasi-static tests suggest that Zpins have negative contribution on the failure strain. The influence of Z-pins on the failure strain was insignificant for compression tests with high off-axis angle.

The failure stress increased with loading rate in all tests for both unpinned and Zpinned samples as shown in Fig.10b. Z-pinning reduces the failure stress for both the shear dominated compression tests and the tension tests. It is worth noting that the reduction of dynamic failure strength is much larger than that observed in quasi-static regime. In the compression tests with high off-axis angle, Z-pinning has no impact on the strength. It can even be argued that the Z-pins helped to increase the strength slightly.

\section{Discussion}

The normal stress and in-plane shear stress in local material coordinate system can be calculated from these off-axis experiments as:

$$
\begin{aligned}
& \sigma_{22}=\sigma_{x x} \sin ^{2} \beta \\
& \tau_{12}=-\sigma_{x x} \sin \beta \cos \beta
\end{aligned}
$$

where $\sigma_{22}$ is the normal stress, and $\tau_{12}$ is the in-plane shear stress in local material coordinate 
system. The failure stresses in local material coordinate system can then be used to assess the inter-fibre failure envelope of the IM7/8552 material. As shown in Fig.11, the failure envelope of unpinned samples expanded with the increase of strain rate. Referring to the three inter-fibre failure modes shown in Fig.3, the Z-pins have negative effect on the strength when loaded in Mode " $A$ " and " $B$ ". In the Mode " $C$ " domain of the stress spectrum, there is no statistically significant influence on the inter-fibre strength from the presence of Z-pinning. Increasing the loading rate applied to Z-pinned laminates leads to an increase in strength on both tension and compression load cases.

The fracture surface from high strain rate experiments has been observed with Scanned Electron Microscopy (SEM). There were remnants of Z-pins on the fracture surface of Z-pinned samples loaded in dynamic tension, as shown in Fig.12 a\&b. It suggests that cracks may have initiated around the epoxy pockets and Z-pins, causing the premature failure of the laminate. A very similar fracture surface was also found for the dynamic compression at $45^{\circ}$, where cracks across the epoxy pocket can be clearly noticed in Fig.12c. Hence, the imperfection caused by Z-pinning, such as the epoxy pocket and fibre waviness [17], was responsible for the reduced in-plane performance under tension and shear dominated stress conditions. The epoxy matrix of composite laminates tends to be more brittle at higher loading rate. Accordingly, laminates may be more sensitive to these imperfections in dynamic tests, resulting in greater reduction in failure stress than that in quasi-static tests.

The dynamic transverse compression tests (Fig.12d) produced a different fracture surface than that in Fig.12 a-c, where broken pins were found on the surface that had been partly pulled out of the laminates. There were multiple cracks developed across the surface where some of these cracks travelled along the epoxy pocket as well, similar to that of the $45^{\circ}$ 
compression in Fig.12c. These cracks developed under a combined effect of the initial crack and the resistance from the Z-pins, as suggested by the failure process recorded in Fig.5. Therefore, the presence of Z-pin was not a source for crack initiation, while the rupturing and pulling out of Z-pins from the inclined crack surface contributed to an enhanced resistance against crack propagation.

The output from this study showed a consistent trend with the off-axis compression tests at quasi-static loading rate [17]. The in-plane performance decreased with the presence of the Z-pins in shear and tension dominated failure modes (Modes " $A$ " and " $B$ " in Fig.3), while was enhanced when loaded at large off-axis angle such as transverse compression(Mode " $\mathrm{C}$ " in Fig.3). Difference in failure modes of Z-pins and the epoxy pockets, was responsible for the change in Z-pin influence. The in-plane failure mechanisms of Zpinned laminates is very complicated and its evolution with strain rate involves many issues including the rate and pressure dependent plasticity of laminate matrix, as well as the complex failure of Z-pins which is also loading rate dependent[12,13].

An effort has been made to study the transverse tensile failure response of Z-pinned laminates for the first time in this work. The effect of Z-pinning on its failure stress, and the underlining failure mechanisms, are found very similar to the compressive failure when dominated by in-plane shear stress. Considerable rate dependence of failure strength can be noticed from the results of current Z-pinned and unpinned samples. The dynamic tension test configuration needs to be further refined to mitigate the large scatters as observed in Fig.11.

\section{Conclusions}


Z-pinning has been found to be detrimental to the inter-fibre strength in shear and tension dominated stress conditions. The reduction of laminate strength was more significant in dynamic tests than that of the quasi-static tests. Z-pinning rupturing and pulling out enhanced the damage resistance of Z-pinned laminates when loaded largely in compression.

The dynamic stiffness of Z-pinned laminates was found to be comparable to that of unpinned ones, due to the increased stiffness of epoxy pocket at high loading rate.

The failure strength of unpinned samples increased with the rise of strain rate.

\section{Acknowledgement}

The financial support from Engineering and Physical Sciences Research Council (EPSRC) of this research through grant No. EP/ M012905/1 is gratefully acknowledged. Data underlying this paper can be accessed through the Cranfield University repository at https://doi. org/10.17862/cranfield.rd.7955918

\section{References}

[1] A. Puck, M. Mannigel, Physically based non-linear stress-strain relations for the interfibre fracture analysis of FRP laminates, Compos. Sci. Technol. 67 (2007) 1955-1964. doi:10.1016/j.compscitech.2006.10.008.

[2] S. Awang Ngah, A.C. Taylor, Fracture behaviour of rubber- and silica nanoparticletoughened glass fibre composites under static and fatigue loading, Compos. Part A Appl. Sci. Manuf. 109 (2018) 239-256. doi:10.1016/j.compositesa.2018.02.028.

[3] S. Sprenger, M.H. Kothmann, V. Altstaedt, Carbon fiber-reinforced composites using an 
epoxy resin matrix modified with reactive liquid rubber and silica nanoparticles, Compos. Sci. Technol. 105 (2014) 86-95. doi:10.1016/j.compscitech.2014.10.003.

[4] R. Sadeghian, S. Gangireddy, B. Minaie, K.T. Hsiao, Manufacturing carbon nanofibers toughened polyester/glass fiber composites using vacuum assisted resin transfer molding for enhancing the mode-I delamination resistance, Compos. Part A Appl. Sci. Manuf. 37 (2006) 1787-1795. doi:10.1016/j.compositesa.2005.09.010.

[5] H. Cui, Y. Li, Failure of composite T-joints in bending with through-the- thickness reinforcement: stitching vs Z-pinning, Key Eng. Mater. 526 (2013) 233-236. doi:10.4028/www.scientific.net/KEM.525-526.233.

[6] K. Dransfield, C. Baillie, Y. Mai, K. Dransfield, C. Baillie, Y. Mai, Improving the delamination resistance of CFRP by stitching - a review, Compos. Sci. Technol. 50 (2006) 305-317.

[7] A.P. Mouritz, Review of z-pinned composite laminates, Compos. Part A Appl. Sci. Manuf. 38 (2007) 2383-2397. doi:10.1016/j.compositesa.2007.08.016.

[8] K.L. Rugg, B.N. Cox, R. Massabo, Mixed mode delamination of polymer composite laminates reinforced through the thickness by z- ${ }^{\circledR}$ bers, Compos. Part A Appl. Sci. Manuf. 33 (2002) 177-190.

[9] T.M. Koh, S. Feih, A.P. Mouritz, Experimental determination of the structural properties and strengthening mechanisms of z-pinned composite T-joints, Compos. Struct. 93 (2011) 2222-2230. doi:10.1016/j.compstruct.2011.03.009.

[10] F. Pegorin, K. Pingkarawat, S. Daynes, A.P. Mouritz, Mode II interlaminar fatigue properties of z-pinned carbon fibre reinforced epoxy composites, Compos. Part A Appl. 
Sci. Manuf. 67 (2014) 8-15. doi:10.1016/j.compositesa.2014.08.008.

[11] H. Cui, Y. Li, S. Koussios, L. Zu, A. Beukers, Bridging micromechanisms of Z-pin in mixed mode delamination, Compos. Struct. $93 \quad$ (2011) 11. doi:10.1016/j.compstruct.2011.06.004.

[12] H. Cui, M. Yasaee, G. Kalwak, A. Pellegrino, I.K. Partridge, S.R. Hallett, G. Allegri, N. Petrinic, Bridging mechanisms of through-thickness reinforcement in dynamic mode I\&II delamination, Compos. Part A Appl. Sci. Manuf. 99 (2017) 198-207. doi:10.1016/j.compositesa.2017.04.009.

[13] H. Cui, M. Yasaee, S.R. Hallett, I.K. Partridge, G. Allegri, N. Petrinic, Dynamic bridging mechanisms of through-thickness reinforced composite laminates in mixed mode delamination, Compos. Part A Appl. Sci. Manuf. 106 (2018) 24-33. doi:https://doi.org/10.1016/j.compositesa.2017.11.017.

[14] D.D.R. Cartié, G. Dell'Anno, E. Poulin, I.K. Partridge, G. Dell'Anno, E. Poulin, I.K. Partridge, 3D reinforcement of stiffener-to-skin T-joints by Z-pinning and tufting, Eng. Fract. Mech. 73 (2006) 2532-2540. doi:10.1016/j.engfracmech.2006.06.012.

[15] P. Chang, A.P. Mouritz, B.N. Cox, Properties and failure mechanisms of z-pinned laminates in monotonic and cyclic tension, Compos. Part A Appl. Sci. Manuf. 37 (2006) 1501-1513. doi:10.1016/j.compositesa.2005.11.013.

[16] C.A. Steeves, N.A. Fleck, In-plane properties of composite laminates with throughthickness pin reinforcement, Int. J. Solids Struct. 43 (2006) 3197-3212. doi:10.1016/j.ijsolstr.2005.05.017.

[17] H. Cui, A.R. Melro, M. Yasaee, Inter-fibre failure of through-thickness reinforced 
laminates in combined transverse compression and shear load, Compos. Sci. Technol. 165 (2018) 48-57. doi:10.1016/j.compscitech.2018.06.011.

[18] K.C. Du, M. Reichel, G. Scharr, Flexural properties of z-pinned composite laminates in seawater environment, (2014) 8343-8354. doi:10.1007/s10853-014-8543-2.

[19] P. Chang, A.P. Mouritz, B.N. Cox, Flexural properties of z-pinned laminates, Compos. Part A Appl. Sci. Manuf. 38 (2007) 244-251. doi:10.1016/j.compositesa.2006.05.004.

[20] S.G. Miller, K.M. Handschuh, M.J. Sinnott, L.W. Kohlman, G.D. Roberts, R.E. Martin, C.R. Ruggeri, J.M. Pereira, Materials, Manufacturing, and Test Development of a Composite Fan Blade Leading Edge Subcomponent for Improved Impact Resistance, Glenn Research Center, NASA, Cleveland, Ohio, 2015.

[21] M. Yasaee, G. Mohamed, A. Pellegrino, N. Petrinic, S.R. Hallett, Strain rate dependence of mode II delamination resistance in through thickness reinforced laminated composites, Int. J. Impact Eng. 107 (2017) 1-11. doi:10.1016/j.ijimpeng.2017.05.003.

[22] H. Cui, D. Thomson, A. Pellegrino, J. Wiegand, N. Petrinic, Effect of strain rate and fibre rotation on the in-plane shear response of $\pm 45^{\circ}$ laminates in tension and compression tests, Compos. Sci. Technol. $135 \quad$ (2016) 106-115. doi:10.1016/j.compscitech.2016.09.016.

[23] J.-L. Tsai, C.T. Sun, Strain rate effect on in-plane shear strength of unidirectional polymeric composites, Compos. Sci. Technol. 65 (2005) 1941-1947. doi:10.1016/j.compscitech.2005.01.013.

[24] H. Koerber, J. Xavier, P.P. Camanho, High strain rate characterisation of unidirectional carbon-epoxy IM7-8552 in transverse compression and in-plane shear using digital 
image correlation, Mech. Mater. $42 \quad$ (2010) 1004-1019. doi:10.1016/j.mechmat.2010.09.003.

[25] H. Zhao, Material behaviour characterisation using SHPB techniques, tests and simulations, Comput. Struct. 81 (2003) 1301-1310. doi:10.1016/s00457949(03)00044-0.

[26] A. Puck, H. Schurmann, Failure analysis of FRP laminates by means of physically based phenomenological models, Compos. Sci. Technol. 58 (2002) 30.

[27] H. Zhao, G.G. Gary, On the use of SHPB techniques to determine the dynamic behavior of materials in the range of small strains, Int. J. Solids Struct. 33 (1996) 13. doi:10.1016/0020-7683(95)00186-7. 


\section{Figure captions}

Fig.1 specimen configuration (a) compression sample; (b) the Z-pin pattern in the laminates; (c) tension sample

Fig.2. Dynamic test setup with Hopkinson bar systems: (a) tension test; (b) compression test;(c) real test system

Fig.3 Typical inter-fibre failure modes

Fig.4. Dynamic failure modes in (a) $45^{\circ}$ tension of unpinned sample; (b) $45^{\circ}$ tension of Zpinned sample;(c) $30^{\circ}$ compression of unpinned sample; (d) $30^{\circ}$ compression of Z-pinned sample

Fig.5. Dynamic failure process in transverse compression of (a) unpinned and (b) Z-pinned sample; rectangles are used to highlight the initial cracks

Fig.6. Comparison of stress-strain data from both DIC and Hopkinson bar theory

Fig.7. Representative axial stress-strain curves at both quasi-static and dynamic loading rate:

(a) $30^{\circ}$ compression; (b) $45^{\circ}$ compression; (c) $60^{\circ}$ compression; (d) $90^{\circ}$ compression; (e) $45^{\circ}$ tension; (f) $90^{\circ}$ tension;

Fig.8. Influence of strain rate and Z-pinning on the stiffness plotted with standard deviation

Fig.9. Axial strain in transverse compression of Z-pinned samples in (a) quasi-static test and (b) dynamic test, with global average strain of 0.03 .

Fig.10. Influence of strain rate and Z-pinning on the (a) strength and (b) failure strain for different stress conditions; plotted with standard deviation. 
Fig 11. Failure envelope in both quasi-static and dynamic loading rate

Fig.12. Dynamic fracture surface of Z-pinned samples, tested at (a) $45^{\circ}$ tension, (b) $90^{\circ}$ tension,

(c) $45^{\circ}$ compression and (d) $90^{\circ}$ compressoin 
2019-04-03

Dynamic inter-fibre failure of unidirectional composite laminates with through-thickness reinforcement

Cui, Hao

Elsevier

Cui H, Yasaee M, Melro AR. (2019) Dynamic inter-fibre failure of unidirectional composite laminates with through-thickness reinforcement. Composites Science and Technology, Volume 176, May 2019, pp. 64-71

https://doi.org/10.1016/j.compscitech.2019.04.004

Downloaded from Cranfield Library Services E-Repository 